Received: 2016.05.31

Accepted: 2016.12 .06

Published: 2017.09.15

Authors' Contribution:
A Study Design
B Data Collection
C Statistical Analysis
D Data Interpretation
E Manuscript Preparation
F Literature Search
G Funds Collection

\section{Cardiac Magnetic Resonance Imaging Using an Open 1.0T MR Platform: A Comparative Study with a 1.5T Tunnel System}

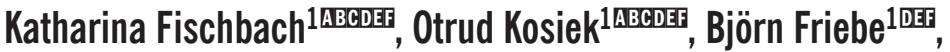 \\ Christian Wybranski ${ }^{10 \mathrm{~F}}$, Bernhard Schnackenburg ${ }^{2 \mathrm{DAF}}$, \\ Alexander Schmeisser ${ }^{3 \mathrm{BDEF}}$, Jan Smid ${ }^{3 \mathrm{BDB}}$, Jens Ricke ${ }^{1 \mathrm{ADE}}$, Maciej Pech ${ }^{1 \mathrm{AGDB}}$ \\ ${ }^{1}$ Department of Radiology and Nuclear Medicine, University Clinic Magdeburg, Magdeburg, Germany \\ ${ }^{2}$ Medical Physics, Philips Healthcare, Hamburg, Germany \\ ${ }^{3}$ Department of Cardiology, University Clinic, Magdeburg, Germany
}

Author's address: Katharina Fischbach, Department of Radiology and Nuclear Medicine, University Clinic Magdeburg, Magdeburg, Germany, e-mail: katharina.fischbach@med.ovgu.de

\section{Summary}

Background: Cardiac magnetic resonance imaging (cMRI) has become the non-invasive reference standard for the evaluation of cardiac function and viability. The introduction of open, high-field, 1.0T (HFO) MR scanners offers advantages for examinations of obese, claustrophobic and paediatric patients.

The aim of our study was to compare standard cMRI sequences from an HFO scanner and those from a cylindrical, 1.5T MR system.

Material/Method:

Fifteen volunteers underwent cMRI both in an open HFO and in a cylindrical MR system. The protocol consisted of cine and unenhanced tissue sequences. The signal-to-noise ratio (SNR) for each sequence and blood-myocardium contrast for the cine sequences were assessed. Image quality and artefacts were rated. The location and number of non-diagnostic segments was determined. Volunteers' tolerance to examinations in both scanners was investigated.

Results: SNR was significantly lower in the HFO scanner (all $\mathrm{p}<0.001$ ). However, the contrast of the cine sequence was significantly higher in the HFO platform compared to the 1.5T MR scanner $(0.685 \pm 0.41$ vs. $0.611 \pm 0.54 ; p<0.001)$. Image quality was comparable for all sequences (all $p>0.05)$. Overall, only few non-diagnostic myocardial segments were recorded: 6/960 (0.6\%) by the HFO and $17 / 960(1.8 \%)$ segments by the cylindrical system. The volunteers expressed a preference for the open MR system $(\mathrm{p}<0.01)$.

Conclusions: $\quad$ Standard cardiac MRI sequences in an HFO platform offer a high image quality that is comparable to the quality of images acquired in a cylindrical 1.5T MR scanner. An open scanner design may potentially improve tolerance of cardiac MRI and therefore allow to examine an even broader patient spectrum.

MeSH Keywords:

Black Blood Sequences • B-SSFP • Cardiac MRI • Cylindrical 1.5T MR System • Open 1.0T MR System

PDF file:

\section{Background}

Cardiac magnetic resonance imaging (cMRI) is widely accepted as the non-invasive reference standard for an accurate and highly reproducible analysis of cardiac function and in vivo myocardial viability. Current guidelines have further strengthened the clinical role of cardiac MRI for a wide variety of myocardial and systemic disorders with a potential cardiac involvement [1,2]. Thus, cardiac MR examinations are increasingly utilized in everyday practice. Currently, cardiac MR examinations are routinely performed in cylindrical MR tunnel systems with a field 
strength of 1.5T. While in the recent years we have seen a trend towards higher field strengths, only after the implementation of advanced multi-source transmission systems can clinical cardiac MRI in cylindrical 3.0T systems be considered equivalent to 1.5T [3]. With the introduction of a "high-field open" (HFO) 1.0T MR platform with a high gradient performance, comprehensive cardiac MR imaging may for the first time be feasible using an open scanner design with image quality and examination speed comparable to $1.5 \mathrm{~T}$.

Thus, the purpose of our study was to perform an intraindividual comparison of standard MR imaging sequences acquired for the evaluation of cardiac morphology and tissue characterisation in a cylindrical $1.5 \mathrm{~T}$ and an open 1.0T MR platform. Cine balanced steady-state free precession (B-SSFP), Tl-weighted (Tl-w) turbo spin echo (TSE), Tl-w black blood (BB) gradient echo (GRE), and T2-w BB TSE sequences were evaluated with regard to 1) image quality, 2) amount of non-diagnostic myocardial segments as well as 3) signal-to-noise-ratio (SNR) at both field strengths and scanner designs in healthy volunteers. Additionally, contrast was assessed for multiphase B-SSFP sequences.

\section{Material and Methods}

\section{Study population}

The study population consisted of 15 healthy volunteers $(5$ male and 10 female, mean age of 34 years, range of 21-70 years; mean height of $170 \pm 7 \mathrm{~cm}$, mean weight of $62 \pm 10$ $\mathrm{kg}$, range $50-83 \mathrm{~kg}$, mean heart rate $65 \pm 7 \mathrm{bpm}$ ). None of the volunteers had a prior history of cardiac events, hypertension or other cardiovascular or pulmonary diseases. All volunteers presented with a normal baseline electrocardiogram (ECG) with sinus rhythm. Written informed consent was obtained from all subjects prior to study inclusion. The study was approved by the local medical ethics committee.

\section{Sequence design and MR examination}

All volunteers were examined both in an open Panorama $\mathrm{HFO}^{\mathrm{TM}}$ (Philips Healthcare, Best, The Netherlands, field strength 1.0Tesla, maximum gradient strength $28 \mathrm{mT} / \mathrm{m}$, slew rate $120 \mathrm{~T} / \mathrm{m} / \mathrm{s}$ ) and a cylindrical 1.5T MR system (Intera 1.5T, Philips Healthcare, Best, The Netherlands, field strength 1.5 Tesla, maximum gradient strength 30 $\mathrm{mT} / \mathrm{m}$, slew rate $150 \mathrm{~T} / \mathrm{m} / \mathrm{s})$. Electrocardiographic gating was performed using a vector-ECG.

At 1.0T, an 8-channel solenoid body coil, and at 1.5T, a dedicated 5-element cardiac-phased array coil was used. The setup in the open 1.0T system is depicted in Figure 1.

The imaging time (defined as the time from the start of the first sequence to the end of the last sequence) and in-room time (defined as the time spent by the volunteer in the MR-suite) were recorded.

Four standard clinical imaging sequences used for characterisation of cardiac function and myocardial tissue were acquired in a $1.5 \mathrm{~T}$ cylindrical MR scanner and compared to an analogous sequence protocol in the open MR platform.

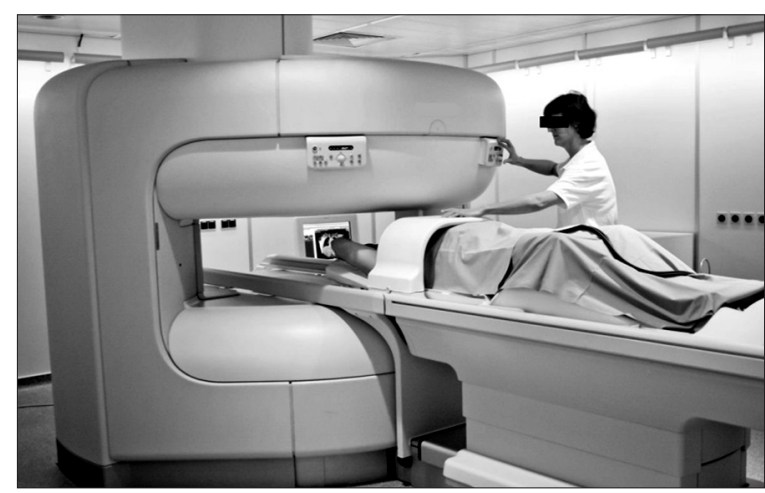

Figure 1. A volunteer placed in the supine position in the 8-channel body coil for diagnostic cardiac MR imaging in an open $1.0 T$ MR system.

The spatial resolution (voxel size) was identical at both field strengths. Functional cardiac MR imaging consisted of 2D multiphase (cine) B-SSFP sequences acquired in three short axial slices (SA: basal, mid-ventricular and apical) of the left ventricle (LV), according to the AHA recommendations (temporal resolution: $54 \mathrm{~ms}$ reconstructed to $27 \mathrm{~ms}$ with view sharing, i.e. 30 reconstructed heart phases). Myocardial tissue was characterised using a Tl-w black blood-prepared segmented GRE sequence (Tl-w BB GRE) and a T2-w black blood-prepared TSE sequence (T2-w BB TSE) as well as a Tl-w TSE sequence without black blood preparation (Tl-w TSE) in three LV SA slices. All sequences were acquired in end-expiration. Sequence parameters for both field strengths are summarized in Table 1.

In order to allow SNR quantification, all SAs were automatically repeated with all RF excitations and gradients disabled ("noise" scans) after a few seconds with identical hardware and software settings (the so-called dynamic scans). No user interaction was required to perform the noise scan, and reconstruction of noise and image data was automatically performed without any sensitivity correction for the surface coils but with the same local coil combination on the commercial reconstruction hardware of the system.

\section{Data analysis}

MR images were transferred to a workstation with the use of a dedicated software application for interpretation and quantitative assessment (Philips View Forum with Cardiac Explorer, Philips Healthcare, Best, The Netherlands). All images were evaluated in consensus by two experienced radiologists (KF 8 years, OK 3 years of experience).

\section{Signal-to-noise ratio (SNR), contrast and contrast-to-noise-ratio (CNR)}

For all 4 sequences, SNR was calculated as follows: $\mathrm{SNR}=$ mean signal intensity (SI) of myocardium/standard deviation (SD) of noise. For each of the four sequences, regions of interest (ROI) were placed in corresponding sections of the interventricular septum in the mid-ventricular SA slice to obtain the mean SI of myocardium at both field strengths. To obtain SD of noise, a large ROI was placed in the "noise" scans in the region of the heart. In addition, for 
Table 1. Sequence parameters for the open $1.0 \mathrm{~T}$ scanner and the $1.5 \mathrm{~T}$ scanner.

\begin{tabular}{lcccc}
\hline \multicolumn{1}{c}{ Parameter } & $\begin{array}{c}\text { B-SSFP } \\
(\mathbf{1 . 0 / 1 . 5 T )}\end{array}$ & $\begin{array}{c}\text { T1-w TSE } \\
(\mathbf{1 . 0 / 1 . 5 T )}\end{array}$ & $\begin{array}{c}\text { T1-w BB GRE } \\
\mathbf{( 1 . 0 / 1 . 5 T )}\end{array}$ & $\begin{array}{c}\text { T2-w BB TSE } \\
\mathbf{( 1 . 0 / 1 . 5 T )}\end{array}$ \\
\hline TR (ms) & $3.3 / 2.7$ & $1000 / 1000$ & $4.7 / 4.3$ & $2000 / 2000$ \\
\hline TE (ms) & $1.64 / 1.35$ & $15 / 15$ & $2.3 / 2.1$ & $80 / 80$ \\
\hline Flip angle a & $55 / 50$ & $90 / 90$ & $40 / 40$ & $90 / 90$ \\
\hline FOV (mm) & $370 / 370$ & $370 / 370$ & $370 / 370$ & $370 / 370$ \\
\hline Matrix & $384 / 384$ & $384 / 384$ & $256 / 256$ & $512 / 512$ \\
\hline Slice thickness (mm) & $8 / 8$ & $8 / 8$ & $8 / 8$ & $8 / 8$ \\
\hline Scan duration (sec)* & $6 / 5$ & $15 / 15$ & $8 / 8$ & $14 / 14$ \\
\hline
\end{tabular}

* Per slice at a heart rate of approximately 65 bpm; TR - repetition time; TE - echo time; FOV - field of view.

the B-SSFP sequence, contrast $(\mathrm{C})$ between the intracavitary blood and the LV myocardium was determined as follows: $\mathrm{C}=($ mean SI of blood - mean SI of myocardium $) /$ mean SI of blood; CNR was calculated as follows: $\mathrm{CNR}=($ mean SI of blood - mean SI of myocardium)/standard deviation (SD) of noise.

\section{Visual assessment of overall image quality}

Overall image quality was rated per sequence and per volunteer on an ordinal, 4-point scale: l=severely impaired, non-diagnostic image quality with severe signal inhomogeneity, 2=moderately impaired image quality with pronounced signal inhomogeneity, however, with diagnostic image quality, $3=$ slightly impaired image quality with mild signal inhomogeneity and good diagnostic image quality, $4=$ excellent image quality with homogenous signal and excellent diagnostic image quality.

\section{Visual assessment of artefacts}

The presence of artefacts was assessed using an ordinal, 5 -point scale $(0=$ none, $1=$ minor, $2=$ moderate, $3=$ major, $4=$ non-diagnostic) for every sequence type and field strength. Furthermore, 16 segments were defined on the three SA slices according to the AHA recommendations [4]. The cardiac apex (segment 17) was not included as no long axis was acquired per protocol. Overall, 960 segments were evaluated per field strength. The location and total number of non-diagnostic myocardial segments was determined in a segment-based assessment according to the AHA's 16-segment model for both field strengths.

\section{Tolerance assessment}

After both examinations were completed, volunteers were again contacted and were asked identical sets of questions regarding feelings of anxiety, claustrophobia or discomfort during the examination both in the open and the cylindrical MR systems. Each of the aforementioned feelings was rated on a four-point, ordinal scale (1: none; 2: a little; 3 : quite a bit; and 4: very anxious/claustrophobic/uncomfortable). Furthermore, they were questioned whether they would consider having such an exam again. To directly compare the two scanner types, the volunteers were asked to express if there was a preference for one of the designs with respect to scan comfort, space in the scanner and perceived safety of the procedure.

\section{Statistical analysis}

In all descriptive statistics, continuous variables are presented as arithmetic mean \pm 1 standard deviation; minimum and maximum values are given in parentheses.

For the assessment of statistical significance, Wilcoxon signed rank tests were performed. Differences were considered statistically significant with a $\mathrm{p}$ value $<0.05$. For statistical analysis, SPSS software, version 21 (Statistical Package for the Social Sciences; SPSS Inc., Chicago, IL) was used.

\section{Results}

The mean imaging time was $14 \pm 1 \mathrm{~min}(12-15 \mathrm{~min})$ and $13 \pm 1 \mathrm{~min}(12-14 \mathrm{~min})$, and in-room time was $23 \pm 1 \mathrm{~min}$ (22-24 min) and 23 $\pm 1 \mathrm{~min}(22-25 \mathrm{~min})$ per volunteer in the open platform and the cylindrical 1.5T MR system, respectively.

\section{Signal-to-noise ratio (SNR) and contrast}

The mean SNR values were significantly lower $(p<0.001)$ in the open, $1.0 \mathrm{~T}$ scanner for all four sequences compared to the standard cylindrical 1.5T MR scanner (Table 2). The contrast of B-SSFP sequences was significantly higher when acquired with the open MR platform $(0.685 \pm 0.41)$ as compared to the cylindrical $1.5 \mathrm{~T}$ MR scanner $(0.611 \pm 0.54)$ $(\mathrm{p}<0.001)$. In contrast, CNR values were significantly higher for the cylindrical $1.5 \mathrm{~T}$ scanner $(73 \pm 21)$, as compared to the open MR platform $(29 \pm 4)(\mathrm{p}<0.001)$.

\section{Visual assessment of overall image quality}

Image quality of all four sequence types did not show a statistically significant difference between the two scanner designs ( $p>0.05)$ (Table 3). 
Table 2. SNR values of standard unenhanced cine and tissue characterization sequences both at an HF0 and a cylindrical 1.5T MR system.

\begin{tabular}{lcccc}
\hline SNR & $\begin{array}{c}\text { Open 1.0T (HF0) MR system } \\
\text { (mean } \pm \text { SD, [range]) }\end{array}$ & \multicolumn{2}{c}{$\begin{array}{c}\text { Cylindrical 1.5T MR system } \\
\text { (mean } \pm \text { SD, [range]) }\end{array}$} \\
\hline B-SSFP & $12 \pm 1.5$ & $(9-15)$ & $32 \pm 10$ & $(23-59)$ \\
\hline T1-w TSE & $67 \pm 18$ & $(25-88)$ & $205 \pm 32$ & $(162-271)$ \\
\hline T1-w BB GRE & $32 \pm 6$ & $(24-43)$ & $79 \pm 16$ & $(48-104)$ \\
\hline T2-W BB TSE & $33 \pm 13$ & $(17-65)$ & $85 \pm 23$ & $(54-140)$ \\
\hline
\end{tabular}

Table 3. Overall image quality ( $1=$ non-diagnostic; 4 excellent image quality) of standard unenhanced cine and tissue characterization sequences both at an HFO and a cylindrical 1.5T MR system.

\begin{tabular}{lcccc}
\hline $\begin{array}{c}\text { Overall } \\
\text { image quality }\end{array}$ & $\begin{array}{c}\text { Open 1.0T (HFO) MR system } \\
\text { (mean } \pm \text { SD, [range]) }\end{array}$ & \multicolumn{2}{c}{$\begin{array}{c}\text { Cylindrical 1.5T MR system } \\
\text { (mean } \pm \text { SD, [range]) }\end{array}$} \\
\hline B-SSFP & $3.8 \pm 0.41$ & $(3-4)$ & $3.6 \pm 0.51$ & $(3-4)$ \\
\hline T1-w TSE & $3.3 \pm 0.59$ & $(2-4)$ & $3.6 \pm 0.51$ & $(3-4)$ \\
\hline T1-w BB GRE & $3.5 \pm 0.64$ & $(2-4)$ & $3.3 \pm 0.49$ & $(3-4)$ \\
\hline T2-w BB TSE & $3.5 \pm 0.64$ & $(2-4)$ & $3.4 \pm 0.51$ & $(3-4)$ \\
\hline
\end{tabular}

Table 4. Visual assessment of artifacts ( $0=$ none; $4=$ non-diagnostic) of standard unenhanced cine and tissue characterization sequences both at an HFO and a cylindrical 1.5T MR system.

\begin{tabular}{lcccc}
\hline $\begin{array}{c}\text { Visual assessment of } \\
\text { artifacts }\end{array}$ & $\begin{array}{c}\text { Open 1.0T (HFO) MR system } \\
\text { (mean } \pm \text { SD, [range]) }\end{array}$ & \multicolumn{2}{c}{$\begin{array}{c}\text { Cylindrical 1.5T MR system } \\
\text { (mean } \pm \text { SD, [range]) }\end{array}$} \\
\hline B-SSFP & $0.3 \pm 0.46$ & $(0-1)$ & $0.8 \pm 1.01$ & $(0-3)$ \\
\hline T1-w TSE & $1.3 \pm 0.72$ & $(0-3)$ & $1.2 \pm 1.01$ & $(0-3)$ \\
\hline T1-w BB GRE & $0.6 \pm 0.91$ & $(0-3)$ & $1.1 \pm 1.06$ & $(0-3)$ \\
\hline T2-w BB TSE & $1.1 \pm 1.23$ & $(0-3)$ & $1.2 \pm 1.26$ & $(0-3)$ \\
\hline
\end{tabular}

\section{Visual assessment of artefacts}

For three (B-SSFP, T1-W BB GRE, T2-w BB TSE) of the four tested sequences, fewer artefacts occurred in the open MR platform, as compared to the standard cylindrical 1.5T MR system (Table 4). The Tl-w TSE sequence was the only sequence with slightly more artefacts in the open scanner (Table 4). However, the difference in artefact occurrence for all four sequences was not statistically significant $(\mathrm{p}>0.05)$.

Non-diagnostic segments occurred primarily in the $\mathrm{T} 2-\mathrm{w}$ BB TSE sequence (5/240 segments, $2.0 \%)$ in the open $1.0 \mathrm{~T}$ platform, with only one non-diagnostic segment for the Tl-w BB GRE sequence (0.4\%).

Using the cylindrical MR tunnel system, 8 of the 240 segments $(3.3 \%)$ were non-diagnostic for the T2-w BB TSE, and 9 of the 240 segments (3.8\%) were non-diagnostic for the Tl-w, BB GRE sequence. The majority of non-diagnostic segments at both field strengths was detected in the basal SA slices (Figures 2-5).

\section{Tolerance assessment}

There was a statistically significant difference between volunteers' perceptions of anxiety, claustrophobia and discomfort between the scanner types in favour of the open 1.0T MR scanner: anxiety - (1.0T vs. 1.5T): $1.1 \pm 0.35$ (range 1-2) vs. $1.8 \pm 0.86$ (range 1-4); claustrophobia (1.0T vs. $1.5 \mathrm{~T}$ ): $1.1 \pm 0.26$ (range $1-2$ ) vs. $1.6 \pm 0.74$ (range $1-3$ ); discomfort (1.0T vs. $1.5 \mathrm{~T}$ ): $1.3 \pm 0.62$ (range $1-3$ ) vs. $1.9 \pm 0.88$ (range $1-4)$, [scale 1-4] $(\mathrm{p}<0.05)$. One volunteer stated that he would not have an MRI scan again in the cylindrical scanner. In a direct comparison, 10 volunteers (67\%) stated that overall they preferred the open scanner, while the remaining $5(33 \%)$ expressed no preference.

\section{Discussion}

In an intraindividual comparison of an adult population our prospective study shows that standard cardiac MRI sequences at an HFO platform offer high image quality and excellent contrast that is comparable to sequences acquired in a cylindrical 1.5T MR scanner. 


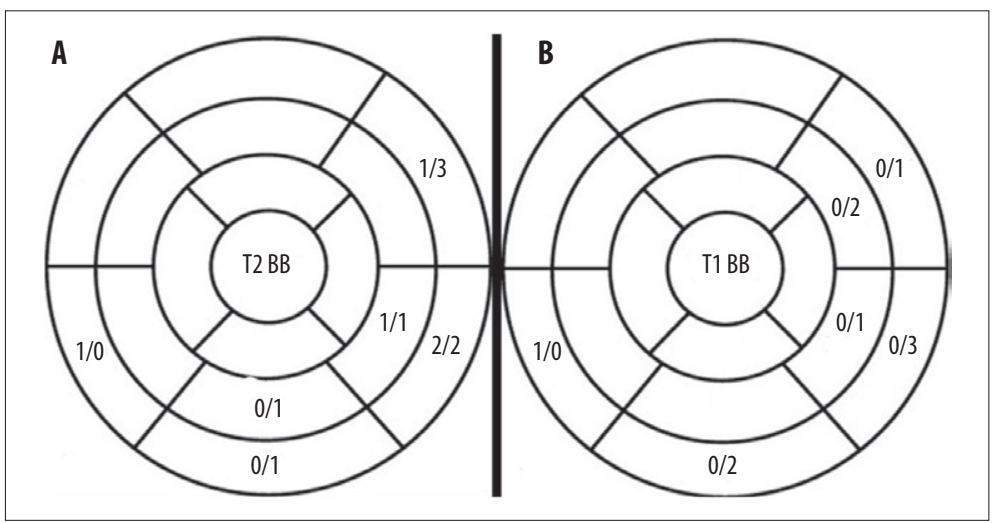

Figure 2. Distribution of non-diagnostic segments in a bull's eye plot according to the 16-segment model of the AHA. Numbers represent the total non-diagnostic segment count in the 1.0T/1.5T scanner for the T2-weighted, black blood, turbo spin echo (A: T2 BB) and for the T1-weighted, black blood, gradient echo (B: T1 BB) sequences. Most nondiagnostic segments occurred in the basal and lateral segments.

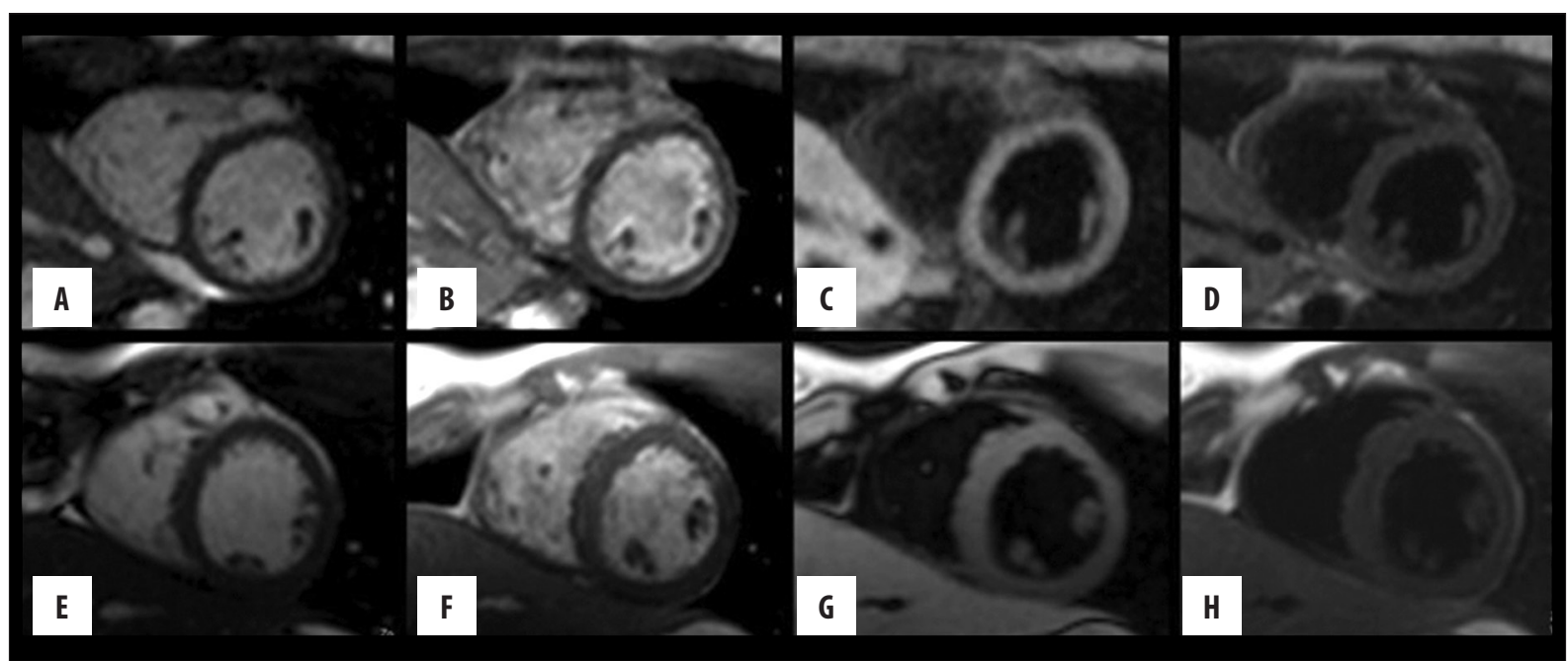

Figure 3. Balanced steady-state, free precession $(A+E)$, T1-weighted turbo spin echo $(B+F)$, T1-weighted black blood gradient echo $(C+G)$ and T2weighted black blood turbo spin echo $(\mathrm{D}+\mathrm{H})$ sequences acquired in mid-ventricular slices both with an open 1.0T MR scanner (A-D) and a standard cylindrical 1.5T MR system (E-H) in the same healthy volunteer. Image quality was rated 4 (excellent) for both scanner designs.

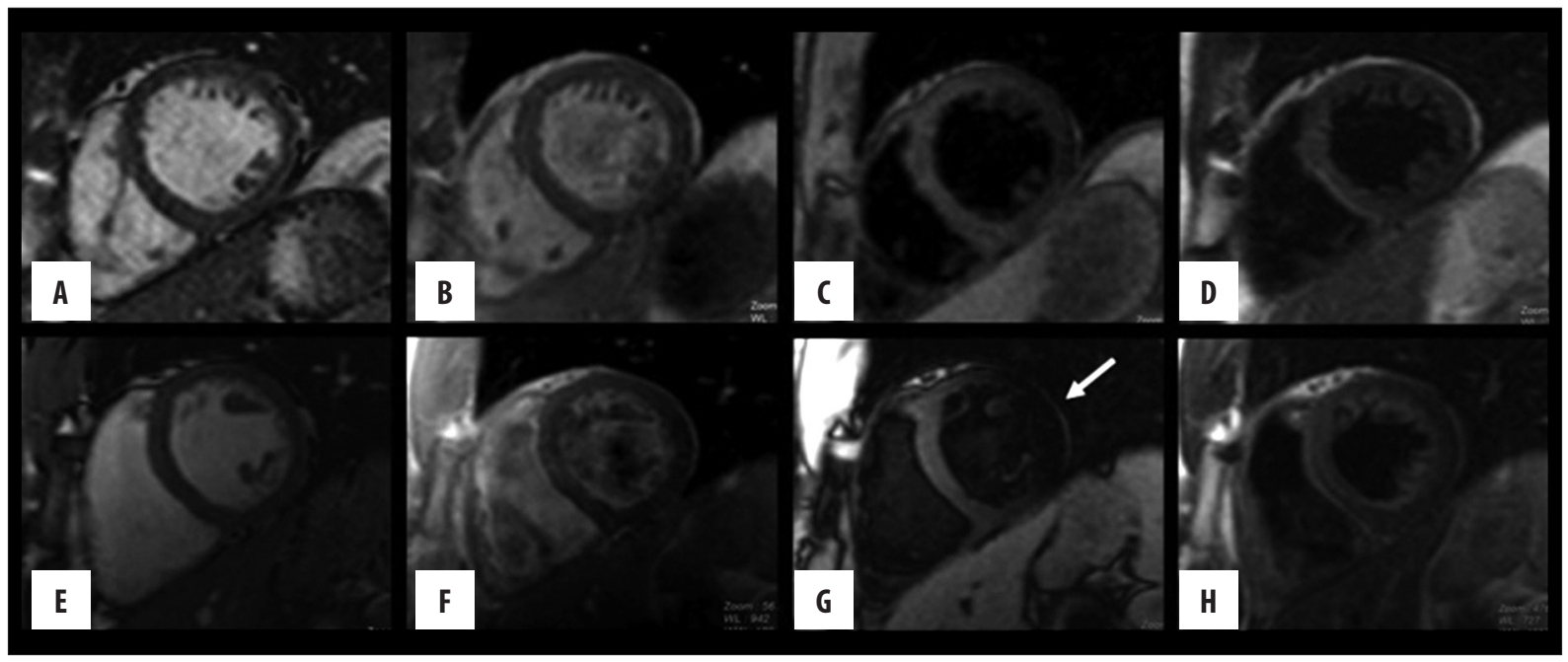

Figure 4. Mid-ventricular, short axis slices obtained using a balanced, steady-state, free precession $(A+E)$, T1-weighted turbo spin echo $(B+F)$, T1-weighted black blood gradient echo $(C+G)$ and T2-weighted black blood turbo spin echo $(D+H)$ sequences at the open 1.0T MR scanner (A-D) and the cylindrical 1.5T MR system (E-H) in a healthy volunteer. Two segments (anterolateral and inferolateral) were nondiagnostic (arrow) in the cylindrical 1.5T system. No non-diagnostic segments were detected using the open 1.0T MR platform. 


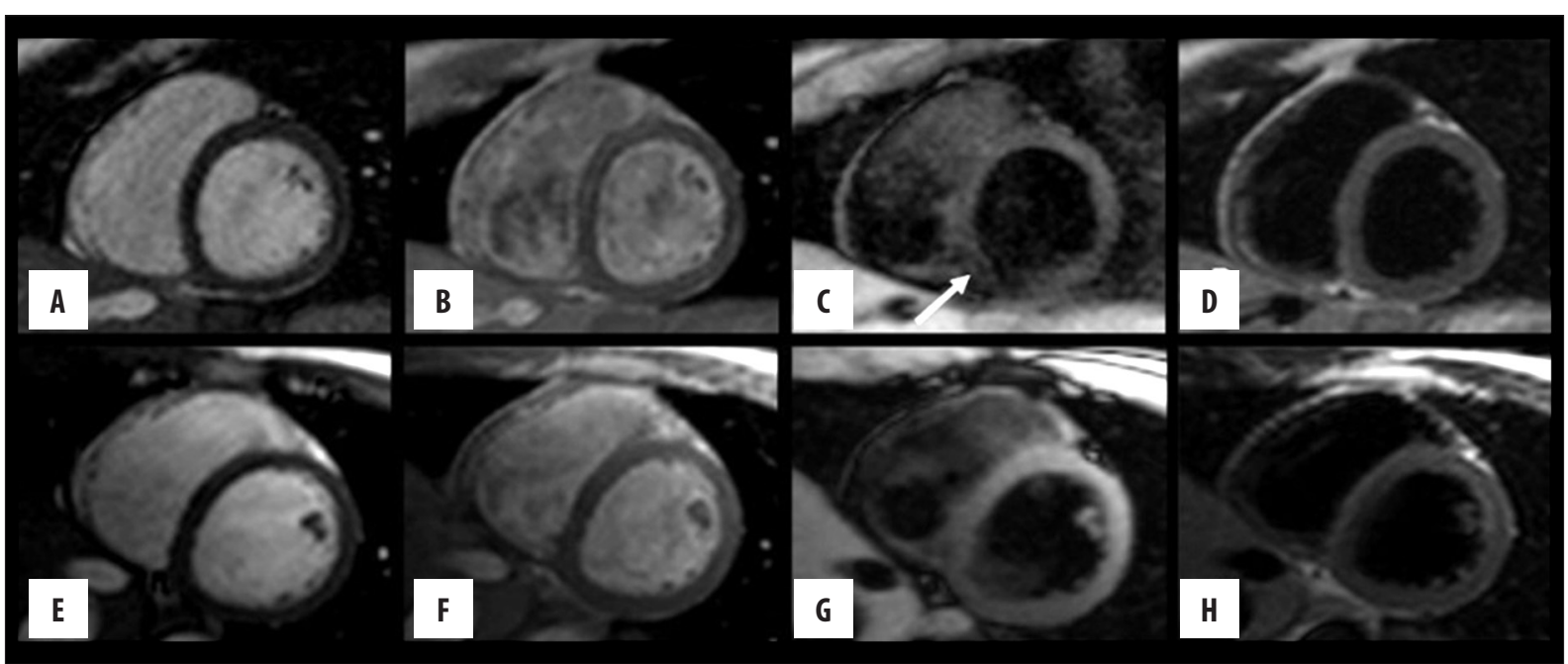

Figure 5. Balanced steady-state free precession $(A+E)$, T1-weighted turbo spin echo $(B+F)$, T1-weighted black blood gradient echo $(C+G)$ and $T 2-$ weighted black blood turbo spin echo $(D+H)$ in the open 1.0T MR scanner (A-D) and in the cylindrical 1.5T MR system (E-H) in a healthy volunteer in whom a non-diagnostic, basal inferoseptal segment was imaged (arrow).

In general, MR scanners can be categorized according to their design in open platforms and cylindrical, tunnel systems. Initially, the term "open" design referred to an open magnet with a C-arm or a vertical design, rather than a horizontal bore. Currently, the term is used for open systems that use two coils of a superconductive magnet as part of a yoke to generate a vertical field ("double donutdesign") [5].

From a clinical viewpoint, truly open, non-tunnel MR systems offer advantages and thus are increasingly used for cardiovascular MR imaging in the clinical setting $[6,7]$. It is well known that patients with claustrophobia or anxiety disorders often fail to complete an MRI examination in tunnel-type systems (incidence from 0.5 to $50 \%$ ) [8]. Interestingly, recent data indicate that over $90 \%$ of patients, who quit an MRI scan in a conventional tunneltype MR machine, can be successfully examined in an open MR platform [9]. This observation is also reflected by our results. Even though we did not include any volunteers with known claustrophobia, the overall rating on comfort/ discomfort during the MR exams was clearly in favour of the open scanner design; one volunteer refused to repeat an exam in the tunnel MR scanner.

In excessively obese patients (WHO obese class II or higher), a patient group prone to cardiovascular disease, echocardiography - as the first line imaging modality in the cardiac work up, is not uncommonly inconclusive due to the limited echo window, and thus cardiac MRI is increasingly called for $[10,11]$. Bucourt et al. was able to show that heavily obese patients who did not succeed to undergo diagnostic computed tomography (CT), standard MR or ultrasound studies were successfully examined in an open MR system [12].

Based on these considerations, Klein et al. assessed a standard cardiac MR protocol in 11 patients using a commercially available open $0.35 \mathrm{~T}$ MR system [13]. They were able to demonstrate that functional imaging of the heart using multiphase (20 heart phases) B-SSFP sequences with an in-plane resolution of $2.3 \times 2.3 \mathrm{~mm}$ was feasible with respect to image quality. Owing to the limited slew rate $(55 \mathrm{~T} / \mathrm{m} / \mathrm{s}$; TE was $2.16 \mathrm{~ms})$, Takizawa et al., utilizing an open 0.7T MR scanner [14], were able to show that the higher field strength, and consequently an increased SNR, allows for the implementation of parallel imaging (acceleration factor 2) to improve temporal (25 vs. 15 heart phases) or spatial resolution (in-plane resolution $2.2 \mathrm{vs}$. $3.5 \mathrm{~mm}$ ) of multiphase B-SSFP cine sequences. However, it is noteworthy that the spatial resolution in both studies fails to comply with today's minimum requirements for cine B-SSFP sequences in the adult and child population [15] asking for an in-plane resolution of $\leq 1.5 \times 3.0 \mathrm{~mm}$ or $2.0 \times 1.5 \mathrm{~mm}$, respectively, with a temporal resolution of $\leq 50 \mathrm{~ms}$. At present, there exists only one other, although retrospective, study by Lu et al. [7] evaluating the clinical efficacy of cardiac MRI in an open 1.0T MR scanner in a paediatric patient population, in accordance with the current recommendations [7]. In contrast, our study prospectively assessed a state-of-the-art cardiac MRI protocol in an adult population by performing an intraindividual comparison of MR imaging in both a cylindrical as well as an open MR platform. All sequences used at a conventional 1.5T tunnel system were optimized for image contrast, spatial and temporal resolution, and used for both systems. Spatial $\left(1 \times 1 \times 8 \mathrm{~mm}^{3}\right)$ and temporal (30 cardiac phases) resolution was kept constant for both scanners. The flip angle was adapted marginally to the field strength $\left(55^{\circ}\right.$ for $1.0 \mathrm{~T}$ vs. $50^{\circ}$ for $1.5 \mathrm{~T}$ ) due to different $\mathrm{Tl}$ values with a difference of only 5 degrees; for that reason, its influence on contrast is negligible. TR/TE values of the B-SSFP sequence vary $(20 \%)$ owing to the different gradient performance. Our data show that overall image quality of multiphase B-SSFP sequences as well as T1- and T2-weighted sequences acquired in an open 1.0T MR platform was high and without a statistically significant difference to an equivalent sequence in the $1.5 \mathrm{~T}$ system. Artefacts, if present in multiphase cine B-SSFP sequences, were minor and none of the segments evaluated were non-diagnostic for both the open 1.0T and the cylindrical 1.5T MR system (artefact score $0.3 \pm 0.46$ vs. $0.8 \pm 1.01$, respectively), which is 
in agreement with previous studies conducted in conventional 1.5T tunnel systems $[16,17]$. In contrast, the blackblood preparation used in $\mathrm{T} 1$ - and $\mathrm{T} 2$-weighted imaging is prone to artefacts $[18,19]$, typically arising in the basal and middle inferior as well as lateral left ventricular segments. They are caused by a signal loss due to reduced surface coil sensitivity with depth of field and also through-plane cardiac motion. In agreement with the literature, artefacts in our study occurred almost exclusively in the segments farthest away from the receiver coils, independent on the MR system configuration. Interestingly, the number of non-diagnostic segments was slightly higher for the cylindrical 1.5T $[\mathrm{n}=17 / 960(1.8 \%)]$ than the open 1.0T MR system $[n=6 / 960(0.6 \%)]$ in our study. This difference could, however, be related to the overall small sample size of our study. Accordingly, Lu et al. [7] reported high image quality for black blood imaging as well as for cine imaging in 95\% of paediatric patients. In three paediatric patients, there was a below-average image quality with cine imaging. This may be explained by the fact that the study sample comprised of young patients with congenital heart disease, with 6 patients under the age of 6 , the youngest 4.5 years old, whereas our adult volunteers were healthy and able to easily collaborate. Additionally, the study by Lu et al. [7] does not offer a direct comparison of image quality with a cylindrical 1.5T MR system, which may have shown image quality to be equally impaired in the respective patients.

An additional advantage of the employed open MR platform is the improved gradient system. While both formerly used systems possess similar amplitudes $(24 \mathrm{mT} / \mathrm{m}$ and $22 \mathrm{mT} / \mathrm{m})$ and a rise time of $0.4 \mathrm{~ms}[13,14]$, the Panorama $\mathrm{HFO}^{\mathrm{TM}}$ system has a higher amplitude $(28 \mathrm{mT} / \mathrm{m})$ as well as a shorter rise time $(0.25 \mathrm{~ms})$. The rise time is a measure of the maximal achievable temporal resolution, and therefore it is critical for functional imaging, i.e. cine B-SSFP sequences. Thus, the rise time translates into the shortest obtainable TR/TE. In our study, TR/TE was 3.3/1.64 for the open Panorama $\mathrm{HFO}^{\mathrm{TM}}$ and 2.7/1.35 for the tunnel system, respectively. In contrast, TR/TE was 4.32/2.16 [13] and 3.8/1.9 [14] in the previous open MR systems.

Previous studies have stressed the importance of a reliable homogeneity of the static magnetic field $\left(\mathrm{B}_{0}\right)$ and gradient fields $[3,16,20]$. As, in general, artefacts increase proportionally with the rising magnetic field strength, the transition from a $1.5 \mathrm{~T}$ to a $1.0 \mathrm{~T}$ scanner is expected to benefit the overall image quality, as shown in our study $[16,17,20]$. The change from a tunnel to an open-magnet design also needs to be considered. There is a small difference in the homogeneity of the $\mathrm{B}_{0}$-field $(0.1 \mathrm{ppm})$ for the open system versus that of the tunnel system (0.07 ppm) (V-RMS values for a 30 $\mathrm{cm}$ sphere). A patient-adapted additional shim procedure (integrated in the sequence preparation phase) was used to improve the homogeneity within the field of view. The results for the B-SSFP sequence show that the $B_{0}$ homogeneity in the open design is more than sufficient.

The measured SNR values in the present study showed a large and surprising difference between the 1.0T- and the 1.5T-system (average factor of 2.5), as the anticipated signal loss was roughly in the range of 30 percent. A cause for the rather large SNR disparity could be the difference in coil hardware. The best available coil setting was used for data acquisition at both field strengths. At 1.5T, the dedicated 5-element cardiac coil was utilized with two flexible elements in the front, directly positioned onto the chest wall, in close proximity to the heart. In contrast, no dedicated cardiac coil exists for the 1.0T MR platform so far. Thus, a rigid, 8-element body coil was used with coil elements at a considerable distance from the thoracic wall. In addition, the visible FOV of the body coil was much larger than the FOV of the cardiac coil, incorporating a significant amount of additional noise in the acquisition, which again impedes SNR values. In principle, it has been reported that the vertically oriented $\mathrm{B}_{0}$-field has advantages regarding the SNR, since it allows for the implementation of the so-called solenoid coils [21]. Most of the elements of solenoid coils enclose the object, whereas the elements of a coil used in a cylindrical MR system form loops at the surface of the object. Due to a higher filling factor, these enclosing coil elements generally offer a better SNR [21,22]. Ham et al. reported that the SNR of a 1.0T vertical field system is comparable to a 1.5T horizontal field system, if differences in hardware (gradients, RF-system, RF coil) or in software can be neglected [23]. However, it is noteworthy that, according to the Rose model on the relationship between background noise and detectable spatial resolution, the minimum SNR value of $\geq 4$ per voxel is required in order to differentiate a single voxel from the background noise [24]. Considering the absolute SNR values obtained in our study, even the minimal SNR value (=12 for B-SSFP) at $1.0 \mathrm{~T}$ is three times larger than this SNR limit.

Besides a sufficient SNR, a good contrast between the LV blood pool and the myocardium is a prerequisite for consistent endocardial border delineation without contrast agents [25]. Even though, in correlation with SNR values, CNR was statistically significantly higher for the cylindrical $1.5 \mathrm{~T}$ system $(73 \pm 21)$ than for the open MR platform $(29 \pm 4)(p<0.001)$ in our study, the contrast of the cine $\mathrm{B}$-SSFP sequence $(0.685 \pm 0.41)$ acquired at the open MR platform was statistically significantly higher than for the cylindrical MR system $(0.611 \pm 0.54, \mathrm{p}<0.001)$. Thus, both scanner designs and field strengths allow for robust image segmentation as well as a reliable detection of wall motion abnormalities.

\section{Limitations}

Due to the limited coil portfolio for the open MR platform, a general body coil was used for cardiac imaging. The application of a dedicated cardiac coil is expected to generate a higher SNR, which is beneficial if higher acceleration factors in parallel imaging are employed. Furthermore, we are aware of the fact that image quality in volunteers tends to be better than in physically compromised patients, and that the study was performed with a limited number of subjects. However, as we directly compared image quality for both scanner types instead of assessing absolute image quality at the open 1.0T MR system alone, this should also be reflected in the clinical practice in patients. Further studies are needed to evaluate the performance of comprehensive cardiac MRI exams including contrast-enhanced sequences, such as perfusion studies and delayed enhancement imaging. 


\section{Conclusions}

State-of-the-art sequences for cardiac function and tissue characterisation performed at an open 1.0T MR platform offer high image quality that is comparable to an examination performed with a cylindrical 1.5T MR scanner in an adult population. The number of non-diagnostic myocardial segments in an open 1.0T MR system is comparable to the standard approach in a closed-bore 1.5T MR magnet. Patient tolerance of cardiac MR imaging may increase in open scanner systems.

\section{References:}

1. Achenbach S, Barkhausen J, Beer M et al: [Consensus recommendations of the German Radiology Society (DRG), the German Cardiac Society (DGK) and the German Society for Pediatric Cardiology (DGPK) on the Use of Cardiac Imaging with Computed Tomography and Magnetic Resonance Imaging]. Rofo, 2012; 184: 345-68 [in German]

2. Hundley WG, Bluemke DA, Finn JP et al: ACCF/ACR/AHA/NASCI/ SCMR 2010 expert consensus document on cardiovascular magnetic resonance: A report of the American College of Cardiology Foundation Task Force on Expert Consensus Documents. J Am Coll Cardiol, 2010; 55: 2614-62

3. Mueller A, Kouwenhoven M, Naehle CP et al: Dual-source radiofrequency transmission with patient-adaptive local radiofrequency shimming for 3.0-T cardiac MR imaging: initial experience. Radiology, 2012; 263: 77-85

4. Cerqueira MD, Weissman NJ, Dilsizian V et al: Standardized myocardial segmentation and nomenclature for tomographic imaging of the heart. A statement for healthcare professionals from the Cardiac Imaging Committee of the Council on Clinical Cardiology of the American Heart Association. Int J Cardiovasc Imaging, 2002; 18: $539-42$

5. Ai T, Morelli JN, Hu X et al: A historical overview of magnetic resonance imaging, focusing on technological innovations. Invest Radiol, 2012; 47: 725-41

6. Suttmeyer B, Teichgraber U, Thomas A et al: Non-invasive ECGtriggered 2D TOF MR angiography of the pelvic and leg arteries in an open 1.0-tesla high-field MRI system in comparison to conventional DSA. Biomed Tech (Berl), 2014; 59: 29-37

7. Lu JC, Nielsen JC, Morowitz L et al: Use of a 1.0 Tesla open scanner for evaluation of pediatric and congenital heart disease: A retrospective cohort study. J Cardiovasc Magn Reson, 2015; 17: 39

8. Hayashi N, Watanabe Y, Masumoto $\mathrm{T}$ et al: Utilization of low-field MR scanners. Magn Reson Med Sci, 2004; 3: 27-38

9. Bangard C, Paszek J, Berg F et al: MR imaging of claustrophobic patients in an open l.0T scanner: Motion artifacts and patient acceptability compared with closed bore magnets. Eur J Radiol 2007; 64: 152-57

10. Garrison RJ, Castelli WP: Weight and thirty-year mortality of men in the Framingham Study. Ann Intern Med, 1985; 103: 1006-9

11. Nagel E, Lehmkuhl HB, Bocksch W et al: Noninvasive diagnosis of ischemia-induced wall motion abnormalities with the use of highdose dobutamine stress MRI: comparison with dobutamine stress echocardiography. Circulation, 1999; 99: 763-70

12. de Bucourt M, Streitparth F, Wonneberger U et al: Obese patients in an open MRI at 1.0 Tesla: Image quality, diagnostic impact and feasibility. Eur Radiol, 2011; 21: 1004-15
13. Klein HM, Meyners W, Neeb B et al: Cardiac magnetic resonance imaging using an open 0.35 T system. J Comput Assist Tomogr, 2007; 31: $430-34$

14. Takizawa M, Goto T, Mochizuki $\mathrm{H}$ et al: Cardiac cine parallel imaging on a $0.7 \mathrm{~T}$ open system. Magn Reson Med Sci, 2004; 3: 45-49

15. Lotz J, Kivelitz D, Fischbach R et al: [Recommendations for utilizing computerized tomography and magnetic resonance tomography in heart diagnosis. 2-Magnetic resonance tomographyl. Rofo, 2009; 181: 800-14 [in German]

16. Strach K, Clauberg R, Muller A et al: Feasibility of high-dose dobutamine stress SSFP Cine MRI at 3 Tesla with patient adaptive local RF Shimming using dual-source RF transmission: Initial results. Rofo, 2013; 185: 34-39

17. Wintersperger BJ, Bauner K, Reeder SB et al: Cardiac steady-state free precession CINE magnetic resonance imaging at 3.0 tesla: Impact of parallel imaging acceleration on volumetric accuracy and signal parameters. Invest Radiol, 2006; 41: 141-47

18. Keegan J, Gatehouse PD, Prasad SK, Firmin DN: Improved turbo spin-echo imaging of the heart with motion-tracking. J Magn Reson Imaging, 2006; 24: 563-70

19. Payne AR, Casey M, McClure J et al: Bright-blood T2-weighted MRI has higher diagnostic accuracy than dark-blood short tau inversion recovery MRI for detection of acute myocardial infarction and for assessment of the ischemic area at risk and myocardial salvage. Circ Cardiovasc Imaging, 2011; 4: 210-19

20. Gutberlet M, Noeske R, Schwinge $\mathrm{K}$ et al: Comprehensive cardiac magnetic resonance imaging at 3.0 Tesla: Feasibility and implications for clinical applications. Invest Radiol, 2006; 41: 154-67

21. Xu S, Gade TP, Matei C et al: In vivo multiple-mouse imaging at 1.5 T. Magn Reson Med, 2003; 49: 551-57

22. Ballon D, Graham MC, Miodownik S, Koutcher JA: Doubly tuned solenoidal resonators for small animal imaging and spectroscopy at 1.5 Tesla. Magn Reson Imaging, 1989; 7: 155-62

23. Ham K, Wartnjes M, Gulpers S: Comparison of image quality between open and cylindrical systems. Proc Intl Soc Mag Reson Med, 2004, 11: 1581

24. Watts R, Wang Y: k-space interpretation of the Rose Model: Noise limitation on the detectable resolution in MRI. Magn Reson Med, 2002; 48: 550-54

25. Thiele $\mathrm{H}$, Nagel E, Paetsch I et al: Functional cardiac MR imaging with steady-state free precession (SSFP) significantly improves endocardial border delineation without contrast agents. J Magn Reson Imaging, 2001; 14: 362-67 